\title{
Peak-locking centroid bias in Shack-Hartmann wavefront sensing
}

\author{
Narsireddy Anugu, ${ }^{1,2 \star ~ P a u l o ~ J . ~ V . ~ G a r c i a, ~}{ }^{1}$ Carlos M. Correia ${ }^{3}$ \\ ${ }^{1}$ Faculdade de Engenharia, Universidade do Porto, rua Dr. Roberto Frias, 4200-465 Porto, Portugal; \\ CENTRA - Centro de Astrofísica e Gravitação, IST, Universidade de Lisboa, P-1049-001 Lisboa, Portugal \\ ${ }^{2}$ School of Physics, Astrophysics Group, University of Exeter, Stocker Road, Exeter EX4 $4 Q L, U K$ \\ ${ }^{3}$ Aix Marseille Univ, CNRS, LAM, Laboratoire d'Astrophysique de Marseille, Marseille, France.
}

Accepted XXX. Received YYY; in original form ZZZ

\begin{abstract}
Shack-Hartmann wavefront sensing relies on accurate spot centre measurement. Several algorithms were developed with this aim, mostly focused on precision, i.e. minimizing random errors. In the solar and extended scene community, the importance of the accuracy (bias error due to peak-locking, quantisation or sampling) of the centroid determination was identified and solutions proposed. But these solutions only allow partial bias corrections. To date, no systematic study of the bias error was conducted. This article bridges the gap by quantifying the bias error for different correlation peak-finding algorithms and types of sub-aperture images and by proposing a practical solution to minimize its effects. Four classes of sub-aperture images (point source, elongated laser guide star, crowded field and solar extended scene) together with five types of peak-finding algorithms (1D parabola, the centre of gravity, Gaussian, 2D quadratic polynomial and pyramid) are considered, in a variety of signal-to-noise conditions. The best performing peak-finding algorithm depends on the sub-aperture image type, but none is satisfactory to both bias and random errors. A practical solution is proposed that relies on the anti-symmetric response of the bias to the sub-pixel position of the true centre. The solution decreases the bias by a factor of $\sim 7$ to values of $\lesssim 0.02$ pix. The computational cost is typically twice of current cross-correlation algorithms.
\end{abstract}

Key words: Instrumentation: adaptive optics - Techniques: high angular resolution - Techniques: image processing

\section{INTRODUCTION}

The Shack-Hartmann wavefront sensor is commonly used to measure the wavefront aberrations in astronomical adaptive optics (Tyson 2015), optical testing (Malacara 2007), ophthalmology (Burns et al. 2014) or microscopy (Booth 2014). It consists of a two dimensional (2D) array of micro lenses. For a plane wavefront incidence, the spots are focused on the optical axis of the each micro lens - the reference centres. For an aberrated wavefront, the imaged spots are displaced from the reference centres. The estimation of the spot displacements between the aberrated and the reference spots allows one to retrieve the incident aberrated wavefront profile (Dai 1996)

Correlation algorithms are used to estimate spot displacements when extended sources are present (cf. Rais et al. (2016) for a recent review). The cross-correlation: a) is op-

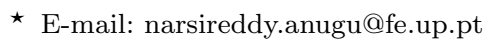

timal at lower signal-to-noise ratios (Vijaya Kumar et al. 1992) and; b) is fast and of simple implementation over other methods such as maximum likelihood (Gratadour et al. 2005) and iterative gradient-based shift estimators (Rais et al. 2016); c) has unitary gain (Gratadour et al. 2010). Cross-correlation is applied to measure image displacements for solar adaptive optics (Wöger \& Rimmele 2009; Löfdahl 2010; Townson et al. 2015), laser guide star elongated spots (Thomas et al. 2008; Basden et al. 2014) and to extended scene wavefront sensing (Poyneer 2003; Robert et al. 2012). The image displacement is computed by cross-correlating a reference ${ }^{1}$ image to the target aberrated sub-aperture image. The correlation algorithm can be implemented either in spatial domain (Löfdahl 2010) or in the Fourier domain (Poyneer 2003; Sidick 2013). In both domains, the image displacement is measured in two steps. In the first step, the

1 Cf. Basden et al. (2014) for several approaches for reference image generation. 
cross-correlation between the reference and the target image is computed. In the second step, a sub-pixel peak-finding algorithm is applied to the correlation image (Poyneer 2003). Commonly used peak-finding algorithms in image registration are 1D parabola fitting (Poyneer 2003; Thomas et al. 2006; Robert et al. 2012), Gauss fitting (Nobach \& Honkanen 2005), centre of gravity, pyramid fitting (Bailey 2003) and 2D quadratic polynomial fitting (Löfdahl 2010). These will be addressed further in the article (cf. Table 1).

Sub-pixel peak-finding in the correlation image is biased towards integer pixels. In adaptive optics, these errors are often referred as systematic bias errors, quantisation errors or sampling errors. Methods for their correction are modelling and a posteriori correction (Wöger \& Rimmele 2009; Löfdahl 2010; Sidick 2013). These approaches are limited because the bias errors depend on: a) modelling; b) the sub-aperture image characteristics; c) the noise level; d) the combination of correlation and the peak-finding algorithms; making it difficult to model - especially in low signal-to-noise conditions.

In the following, the bias problem of centroid algorithms is addressed. In Sec. 2 the methods used are presented, including a novel algorithm for bias error reduction. The results on the bias performance of several peak-finding algorithms are presented in Sec. 3.1. It is found that no algorithm is simultaneously satisfactory for both bias and random errors. The results on the proposed solution to the bias error are presented and discussed in Sec. 3.2. In Sec. 4 we conclude by recalling the main ideas.

\section{METHODS}

\subsection{Current peak-finding methods}

Consider the reference $\left(I_{0}\right)$ and sub-aperture $\left(I_{\mathrm{S}}\right)$ images, with size $N \times N$ pix $^{2}$. The cross-correlation in the image domain $(C)$, is given by

$C[m, n]=\sum_{i=1}^{N} \sum_{j=1}^{N} I_{\mathrm{S}}[i+m, j+n] I_{0}[i, j]$,

The image displacement in integer pixels is determined from the correlation maximum location, which is at the pixel $\left(x_{0}, y_{0}\right)$. The sub-pixel image displacement $\left(s_{x}^{\prime}, s_{y}^{\prime}\right)$ is estimated by applying 2D centroid algorithms (cf. Table 1 and Sec. 1) to the correlation map $C[m, n]$. In most algorithms only five pixels are used: $\left(x_{0}, y_{0}\right),\left(x_{0}-1, y_{0}\right),\left(x_{0}+1, y_{0}\right)$, $\left(x_{0}, y_{0}-1\right)$ and $\left(x_{0}, y_{0}+1\right)$. For the $2 \mathrm{D}$ quadratic polynomial fit nine pixels instead of five are required for the estimation of the six coefficients. The estimation of $s_{y}^{\prime}$ is analogous to $s_{x}^{\prime}$.

The measured displacement $s^{\prime}$ (in a given direction $x$ or $y$ ) by the centroid algorithms is related to the real displacement $s$ by

$s^{\prime}=s+\beta+\epsilon$,

where $\beta$ is the bias error and $\epsilon$ the noise error. As referred in the Introduction these algorithms have systematic errors, the bias error $\beta$ exhibits a "sinusoidal" variation with an exact shape depending on $\epsilon$, image and centroid algorithm. In Fig. 1 an example of this bias is presented, in the absence of noise, for the cross-correlation algorithm (Eq. 1).

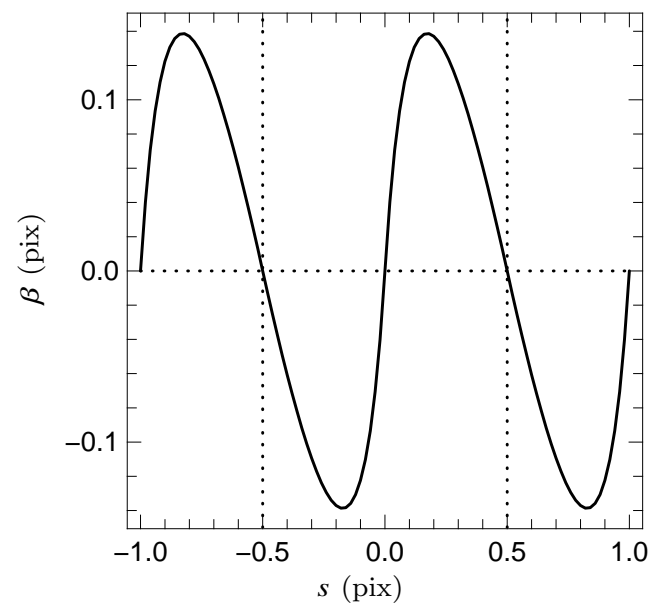

Figure 1. Bias error for a point source with centre of gravity centroid algorithm versus $s_{X}$. The shift vector is $\vec{s}=[s, s]^{T}$, i.e. $s_{x}=s$.

The origin of the bias is well known in the strain measurement community, it is due to the transfer function of the centroid algorithm (e.g. Schreier et al. 2000). For example, the transfer function of the linear interpolation is not unitary but a complex number. Its module and phase changes with interpolation position. Therefore a bias in intensity and shift when an interpolation is made (cf. Schreier et al. 2000, for details).

In the presence of noise $\epsilon$, the bias error $\beta$ in Eq. 2 is estimated by taking the average of a large number of realizations, assuming that $s$ is constant for the number of realizations. The noise error is then significantly reduced and Eq. 2 becomes

$\left\langle s^{\prime}\right\rangle \simeq s+\beta$,

where the \langle\rangle denotes average.

The noise error $\epsilon$ is estimated by the root-mean-square (RMS) deviation of the random sample of realizations

$\epsilon \simeq \sigma=\sqrt{\frac{\sum_{i=1}^{n}\left(s^{\prime}-\left\langle s^{\prime}\right\rangle\right)^{2}}{n}}$,

with $n$ the number of realizations.

\subsection{Window shift peak-finding algorithm}

In the standard approach, the sub-pixel peak centre is determined by directly applying a peak-finding algorithm of Table 1. In this work, a method to reduce the bias error in the peak-finding is proposed. A similar method was previously applied in the context of particle image velocimetry (Gui \& Wereley 2002), but to our knowledge, it is presented for the first time in the context of adaptive optics.

It is a two-step method: a) coarse search; b) fine search. In Algorithm 1 the pseudo-code of the method is presented. In the first step (coarse search, lines 2 to 4 ) the integer pixel maximum location $\left(x_{0}, y_{0}\right)$ is found. In the second step, (fine search, lines 5 to 19 ) an image region of interest ( $I_{\mathrm{ROI}}$, cf. line 12) is interpolated from the sub-aperture image $I_{\mathrm{S}}$. The interpolation is made with the same sampling as the original image. At each iteration, the interpolation is done at 
Table 1. Sub-pixel $\left(s_{x}^{\prime}, s_{y}^{\prime}\right)$ peak-finding algorithms. The estimate of $s_{y}^{\prime}$ is obtained in an analogous fashion to $s_{x}^{\prime}$.

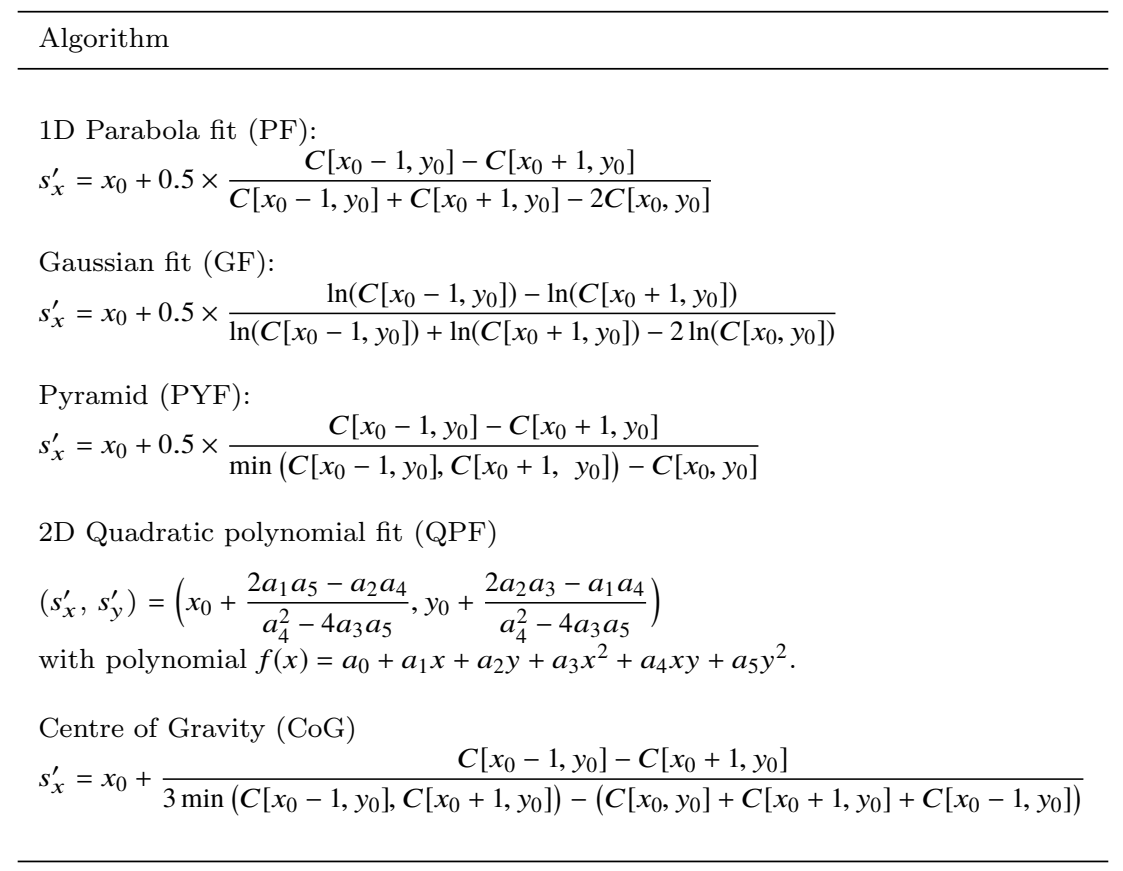

changing fractional initial positions $\delta$ (cf. line 7). Then the correlation between the reference $I_{0}$ and each $I_{\text {ROI }}$ is computed (cf. line 13). The sub-pixel displacements $s^{\prime}$ are then obtained using the peak-finding algorithms of Table 1 (cf. line 16). These sub-pixel displacements are then corrected by the step $\delta$ introduced during the interpolation (cf. line 17). This process is repeated $K$ times, with varying $\delta$ (cf. line 7 ). Because $K$ correlations took place, $s^{\prime}$ is a vector of $K$ elements. The individual displacements $s^{\prime}[k]$ are affected by the bias $\beta$. This bias is "sinusoidal" and anti-symmetric, with period 1 pix, as referred in Fig. 1 . The algorithm then takes the average of all $K$ displacements (cf. line 19), which reduces the bias approximately proportional to $K$.

For computational efficiency the cross-correlation $C$ is not computed in all pixels but only for a sub-image of size $5 \times 5$ pix $^{2}$ centred in the maximum, generating a cropped version of the cross correlation: $C_{5}$. Simulations show that the centroid algorithms behave similarly for $C$ and $C_{5}$.

The combination of the original pixel grid based conventional correlation (in a large field of view) and a sub-image grid correlation within a small field of view, warrants a high dynamic range shift determination to the algorithm.

\subsection{Synthetic sub-aperture images}

Four types of sub-aperture image models of relevance for astronomical adaptive optics were used: a) a point source diffracted spot; b) a laser guide star elongated spot (Schreiber et al. 2009); c) a crowded field image; d) a solar photosphere image (Löfdahl 2010). The simulation of the point source and the laser guide star are realized using $2 \mathrm{D}$ Gaussian profiles (circular $2 \times 2$ pix $^{2}$ and elliptical $3 \times 6$ pix $^{2}$ with a $45^{\circ}$ rotation angle, respectively). The crowded field sub-aperture images are obtained by shifting and adding circular Gaussian profiles of varying intensity. To model the sub-aperture solar photosphere image, a Swedish Solar Telescope solar granulation image is used ${ }^{2}$. All the sub-aperture images are Nyquist sampled and have a size of $16 \times 16$ pix $^{2}$. The synthetic sub-aperture images are presented in Fig. 2.

The synthetic image shifts $(s)$ due to atmospheric tilts are generated as follows. For the point source, laser guide star and crowed field the shifts $s$ are directly applied to the Gaussian profiles. The original solar image has a factor of 10 larger sampling than the one used for the sub-aperture images. The original image is shifted and blurred to the target Nyquist resolution by convolving it with a PSF. The resulting image is binned to generate a $16 \times 16 \mathrm{pix}^{2}$.

Due to the extended and low contrast nature of the solar image, the cross-correlation algorithm is slightly adapted. The mean intensity is subtracted from the reference and subaperture solar images because their linear intensity trend (low contrast) can shift the correlation centre from its correct position (Löfdahl 2010).

Noise is added to the synthetic sub-aperture images. For all images a Gaussian read-out-noise $\left(\sigma_{\mathrm{R}}\right)$ of $1 \mathrm{e}^{-} \mathrm{pix}^{-1}$ is assumed, in line with new generation detectors (Finger et al. 2014; Feautrier et al. 2016). Each synthetic image was generated with counts in each pixel following Poisson statistics. The total image signal-to-noise ratio (SNR) is calculated as

$$
\mathrm{SNR}=\frac{N_{\mathrm{e}}}{\sqrt{N_{\mathrm{e}}+\sigma_{\mathrm{R}}^{2} N_{\mathrm{P}}}},
$$

where $N_{\mathrm{e}}$ and $N_{\mathrm{P}}$ are the total number of electrons and pixels in the sub-aperture image. For reference, $N_{\mathrm{e}}=5 \times 10^{3} \mathrm{e}^{-}$, corresponds to a 9.5 magnitude $\mathrm{H}$-band star with integration

2 http://www.isf.astro.su.se/gallery/ 


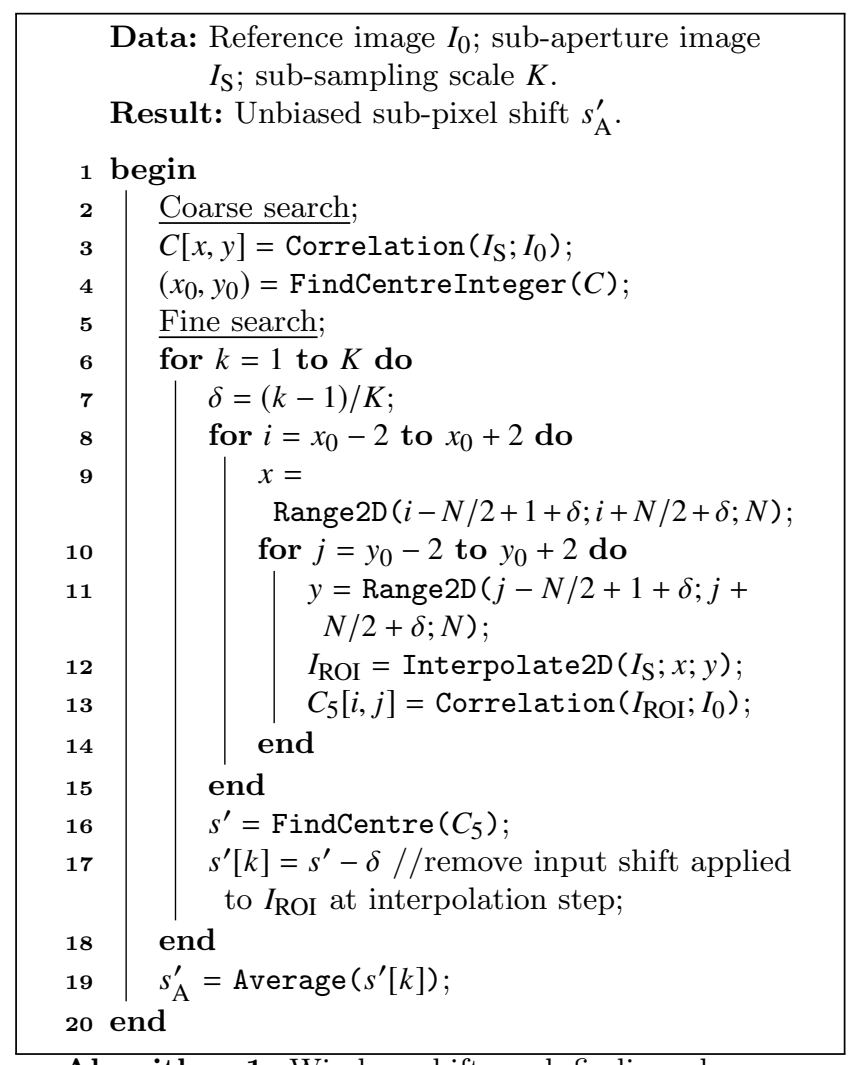

Algorithm 1: Window shift peak-finding algorithm. The function FindCentre is one of the peak-finding algorithms presented in Tab. 1 . The function Correlation $\left(I_{S} ; I_{0}\right)$ is given in Eq. 1. The function FindCentreInteger $(C)$ determines the integer pixel where the maximum of $C$ is located. The function Interpolate2D $\left(I_{\mathrm{S}} ; x ; y\right)$ extracts an interpolated image $I_{\text {ROI }}$ from $I_{\mathrm{S}}$ at grid array locations $x$ and $y$. The function Range2D $(a, b, N)$ creates a square $2 \mathrm{D}$ mesh from $(a, a)$ to $(b, b)$, with $N^{2}$ pixels. In Section 2.2 the algorithm is explained in detail. The $I_{\text {ROI }}$ has image dimensions as $I_{0}$.

time of $10^{-2} \mathrm{~s}$, when a $9 \times 9$ lenslet and a $8 \mathrm{~m}$ class telescope considered. For the solar image case $N_{\mathrm{e}}=5 \times 10^{4} \mathrm{e}^{-}$ corresponds to a $\mathrm{SNR}=104$.

\section{RESULTS AND DISCUSSION}

\subsection{Performance of current peak-finding methods}

\subsubsection{No noise case}

To study the bias, synthetic sub-aperture images displaced horizontally at known positions $s$ are generated. In this section, the sub-aperture images have no noise. The positions $s$ varied from -1 pix to 1 pix, in steps of 0.05 pix. For each synthetic image, the correlation centres $s^{\prime}$ are computed with the conventional cross-correlation as described in Sec. 2.1. Then the peak-finding algorithms of Table 1 are applied to estimate the position $s^{\prime}$. The biases are then simply $\beta=s^{\prime}-s$. For completeness, the bias is also presented for the determination of $s^{\prime}$ with the centre of gravity algorithm directly (i.e. without the correlation) in the sub-aperture images point source and elongated laser guide star. The results are presented in Fig. 3, for the four types of sub-aperture images.

It is found that the bias are anti-symmetric $(\beta(-x)=$ $-\beta(x))$ for all sub-aperture images as expected. It is periodic for the point source, laser guide star and crowded field images. But not periodic for the solar image. This nonperiodicity is due to the extended scene nature of the image. When a shift is applied different parts of the image enter the field of view of the sub-aperture. Therefore the solar image does not have $\beta=0$ pix at $s= \pm 0.5$ pix. The exact shape of the bias curve depends on the centroid algorithm and also on the nature of the image. In the $[-0.5,0.5]$ pix interval the bias extreme values are located approximately at $s= \pm 0.25$ pix for all images except the laser guide $\operatorname{star}^{3}$.

Sharp transitions are observed for extended subaperture images at pixel positions $s= \pm 0.5$ pix. This is due to the shift vector being diagonal: $\vec{s}=[s, s]^{T}$, which translates in the peak of the correlation being "split" into two diagonal pixels. When the shift $s$ is, e.g. 0.4 pix, the brighter pixel is in the lower left one and the bias is negative. When the shift is e.g. 0.6 pix, the brighter pixel is the top right and the bias is positive.

The best performing centroid algorithm depends on the image: a) for the point source and crowded field, it is the Gaussian fit; b) for the solar image it is 2D quadratic polynomial; c) for the laser guide star it is the centre of gravity. The reason for this behaviour is the matching of the algorithm to the actual shape of each image correlation centre (e.g. point source and crowded field are generated with circular Gaussians).

\subsubsection{Varying signal-to-noise ratio}

The centroid algorithms' performance was tested in varying SNR conditions. For each SNR, 500 random realizations are generated at an input shift vector $\vec{s}=\left[s_{\max }, s_{\max }\right]^{T}$ where the bias is approximately maximum: a) $s_{\max }=0.25$ pix for the point, crowded field and solar images; b) $s_{\max }=0.4$ pix for the laser guide star image. Note that the exact maximum location depends on the algorithm and therefore $s_{\max }$ is approximate. The input shifts are measured by applying the conventional cross-correlation (Eq. 1) and centroid algorithm (cf. Table 1). The bias and SNR are computed using Eq. 3 and Eq. 4, respectively. The SNR is varied via $N_{\mathrm{P}}$ in the sub-aperture images.

The results are presented in Fig. 4. The first column presents the RMS centroid error $(\sigma)$. It is computed by the RMS of $s^{\prime}$. It decreases with SNR as expected. The direct image centre determination via the Centre of Gravity algorithm has a worse behaviour for the point source and laser guide star than the correlation algorithms. The superiority of the correlation with respect to $\sigma$ is well known in the literature (e.g. Thomas et al. 2006). Intuitively it is expected because of the noise smoothing and shape matching. All correlation algorithms have a similar behaviour.

3 The shape of the $\beta$ curve for the laser guide star is due to applying shift $\vec{s}=[s, s]^{T}$ along the diagonal of the image. For a horizontal shift, it would have a similar shape as the point source and crowded field. 

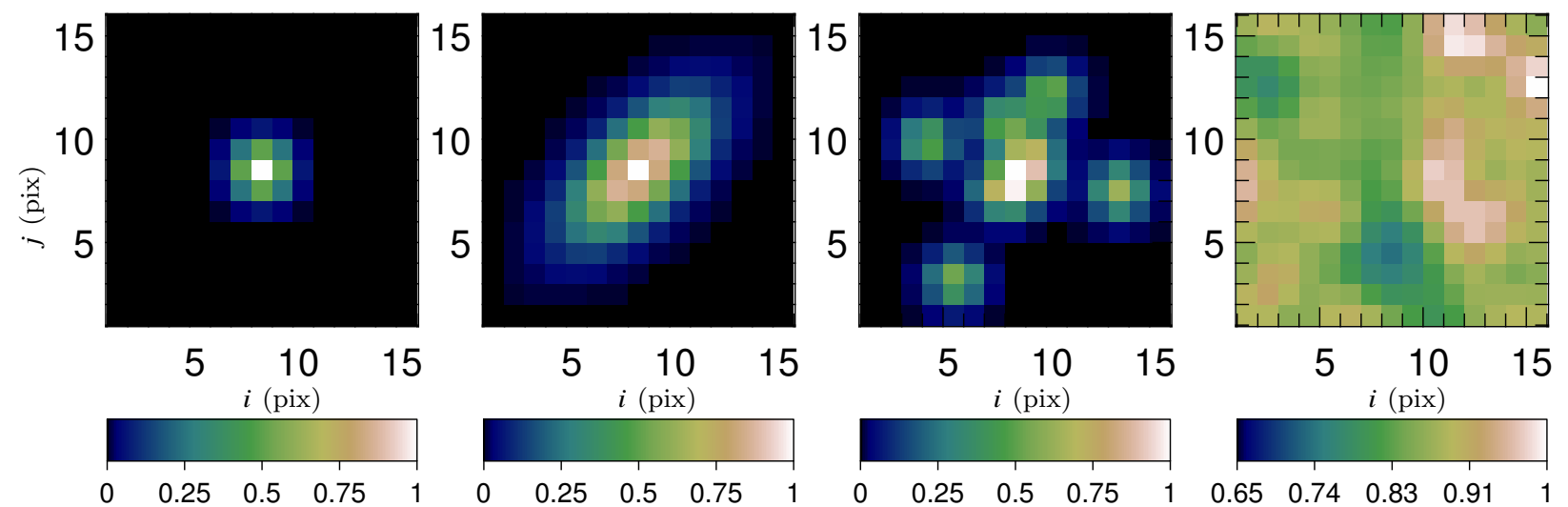

Figure 2. Synthetic Shack-Hartmann sub-aperture images. From left to right: a) the point source; b) the elongated laser guide star (LGS); c) the crowded field; d) the solar photosphere. Colorbars indicate contrast levels.
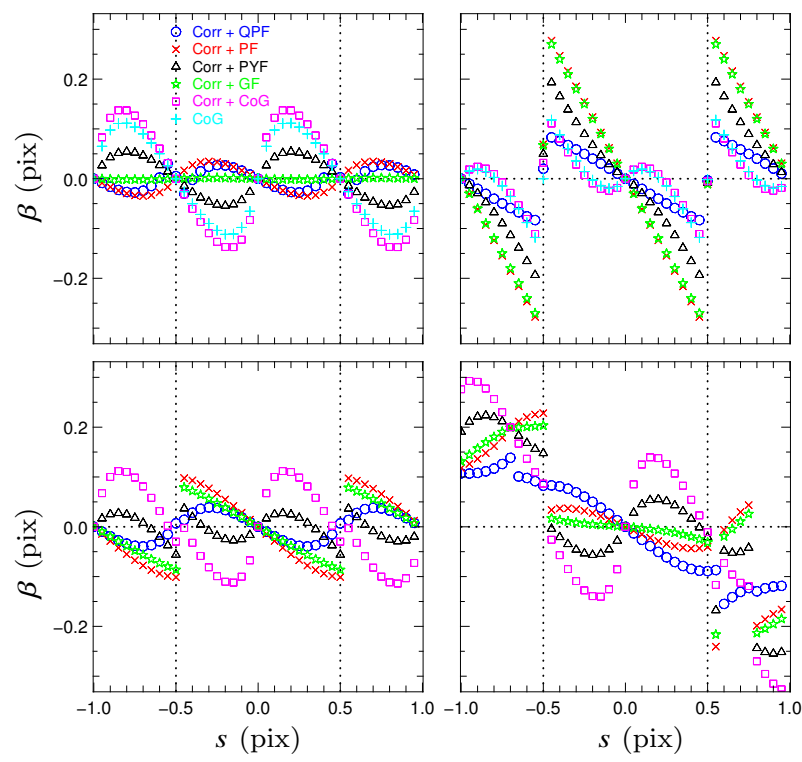

Figure 3. Bias errors for various peak-finding algorithms and sub-aperture images. The algorithm colour/symbol legend is presented at the top left panel, cf. Table 1 for abbreviations translation. The "Corr +" label is used when the algorithm is applied to the correlation image. Sub-aperture images are: point source (top left); laser guide star (top right); crowded field (bottom left); and solar photosphere (bottom right). The shift vector is $\vec{s}=[s, s]^{T}$. The top left (point source) and right (laser guide star) images also include the results for direct application of the Centre of Gravity algorithm to the sub-aperture images, plus signs labelled "CoG".

The centre column of Fig. 4 shows that $\left|\left\langle s^{\prime}\right\rangle-s\right|$ increases to an asymptotic value, the bias error $\beta$. $\left|\left\langle s^{\prime}\right\rangle-s\right|$ is not constant because at low SNR the effective image shape changes. Intuitively the low value of $\left|\left\langle s^{\prime}\right\rangle-s\right|$ at low SNR can be explained by a pure noise image, for which the bias is expected to be zero. The large values of $\left|\left\langle s^{\prime}\right\rangle-s\right|$ for the low SNR of the solar image (centre column, bottom row of Fig. 4) are due to the large variance of $\left|\left\langle s^{\prime}\right\rangle-s\right|$ for this sub-aperture image. The large bias for the laser guide star in comparison to others is caused by the shape of the correlation peak, which is elongated and oriented $45^{\circ}$ rotation angle.

The right column of Fig. 4 plots $\left|\left\langle s^{\prime}\right\rangle-s\right|$ versus $\sigma$.
The dashed line is the imaginary curve $\left|\left\langle s^{\prime}\right\rangle-s\right|=\sigma$. Points above the curve show a bias error larger than the centroid error. Typically the bias error is larger than the noise error for SNR larger than 10, except for the solar case where it becomes important for SNR larger than 200.

\subsection{Performance of the window shift method}

In this section the performance of the algorithm introduced in Section 2.2 is presented, initially for a fixed SNR and then for a varying SNR.

\subsubsection{Fixed signal-to-noise ratio}

Fig. 5 shows the comparison of the window shift method with the conventional cross-correlation algorithm. One of the worst performing centroid algorithms - the centre of gravity - was used. The SNR conditions are the same as in Section 3.1.1. The sampling factor is $K=5$. The window shift method drastically reduces the bias. For the solar image the final bias is larger, because the shift will include pixels from the edge of the image that are not present in the reference image.

\subsubsection{Varying sampling factor $K$}

The effect of the sampling factor $K$ in reducing the bias $\beta$ of the centroid algorithm is presented in Fig.6. The setup is the same as Section 3.2.1, except for the sampling factor $K$, which varied. The centre of gravity algorithm was used considering its better performance against lower SNR for point, crowded field and solar images.

The bias $\beta$ strongly decreases with the sampling factor $K$. It approximately follows a $\propto K^{-1}$ relation. For sampling factors $K>5-6$ no significant improvement is observed. This behaviour is similar to the one observed by Gui \& Wereley (2002) in a different context.

\subsubsection{Varying signal-to-noise ratio}

The performance of the window shift method as a function of SNR is presented in Fig. 7. The same setup as the one 

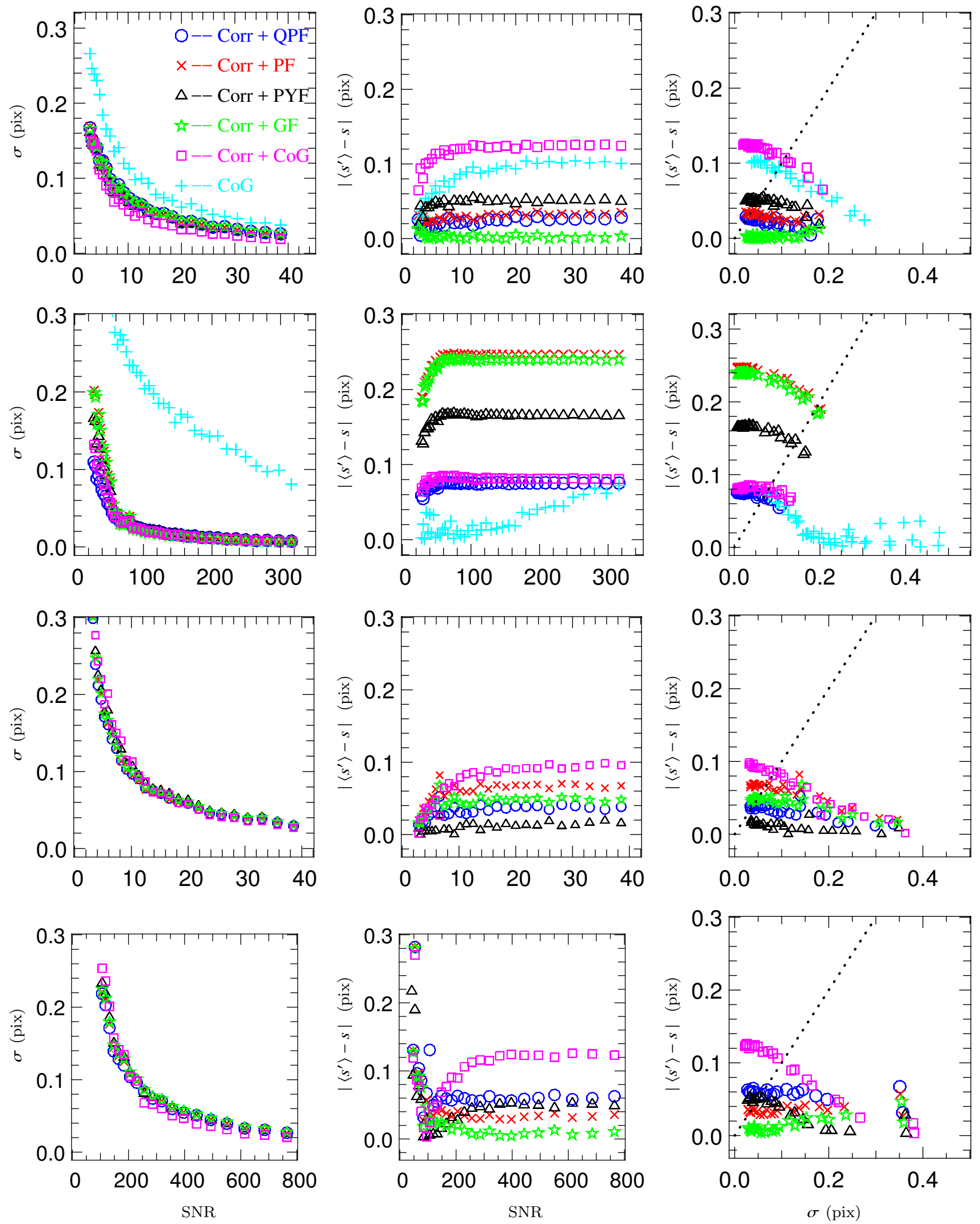

Figure 4. Performance of centroid algorithms with SNR. Left column: RMS centroid error $\sigma$; centre column: $\left|\left\langle s^{\prime}\right\rangle-s\right|$, which coverages to bias error $\beta$ at high SNR; right column $\left\langle s^{\prime}\right\rangle-s$ versus $\sigma$. The dotted line in the right column traces $\left\langle s^{\prime}\right\rangle-s=\sigma$. The rows from top to bottom are: point source, laser guide star, crowded field, solar image, respectively. The dotted line in the last column is where bias error and random error is equal. The data points above this dotted line indicate bias error domination over random error. 

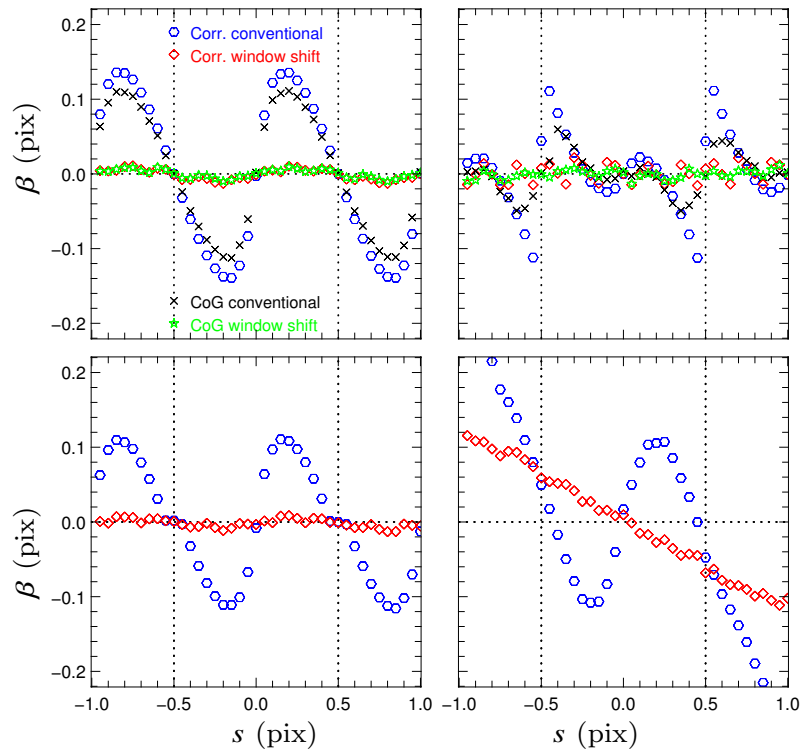

Figure 5. Bias errors of the window shift method in comparison with the conventional approach. The algorithm legend is presented at the top left panel. Sub-aperture images are: point source (top left); laser guide star (top right); crowded field (bottom left); and solar photosphere (bottom right). The shift vector is $\vec{s}=[s, s]^{T}$. The top row also includes the performance for direct sub-aperture images (labelled CoG).

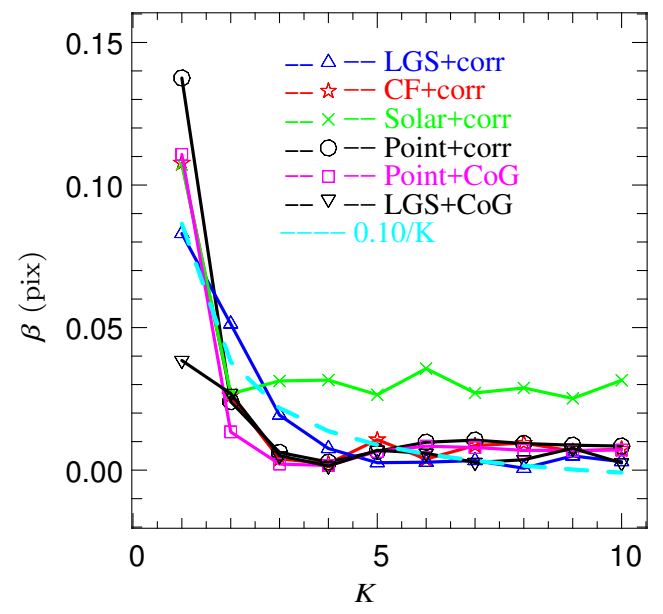

Figure 6. Performance of the window shift method as a function sampling factor $K$. The curves are for: point source, laser guide star (LGS), crowded field (CF) and solar image. The dashed curve depicts the function $\beta(K)=0.1 / K$.

presented in Section 3.1.2 is used (e.g. $s$ position). The sampling factor is $K=5$. The residual errors are well below 0.05 pix for all SNR and sub-aperture image type.

The performance is similar to the windowed, adaptive thresholding centre of the mass method of Townson et al. $(2015)^{4}$.

${ }^{4}$ Note that a different SNR metric is used in Townson et al. (2015), i.e. in their Figure 7 a SNR of 20 corresponds to an SNR 100 in our Figure 7.
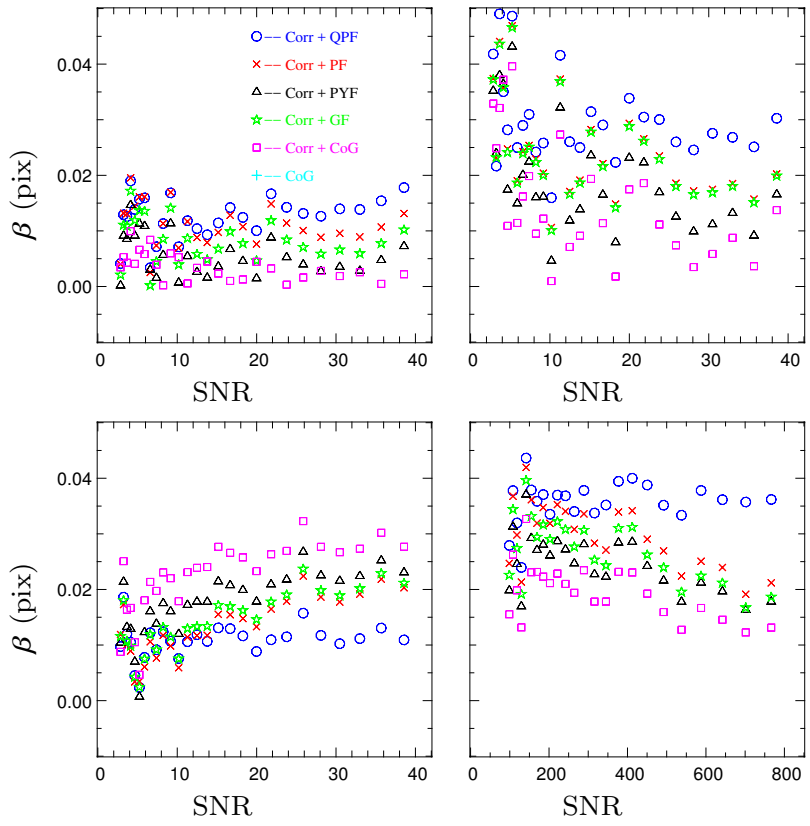

Figure 7. Residual bias errors for window shift method as a function of SNR: point source (top left); laser guide star (top right); crowded field (bottom left); and solar photosphere (bottom right).

\subsubsection{Computational efficiency}

Several number of factors can influence the total execution time of the algorithm, importantly, the sub-aperture window size, the efficiency of programming (ex. multithreading), the performance of hardware and the programming language. Therefore only the relative computational efficiency was computed. The proposed window shift method is slower in comparison to the conventional algorithm by a factor of 2.4 and 3.6 for $K=3$ and $K=5$, respectively. For larger sub-apertures, the computational time ratio is reduced because the window shift method uses a fixed and small correlation sub-image $C_{5}$. If the sub-aperture size is increased from $16 \times 16$ pix $^{2}$ to $32 \times 32$ pix $^{2}$ the window shift method is slower by a factor of 1.4 and 1.8 for $K=3$ and $K=5$, respectively.

\section{CONCLUSIONS}

A systematic study of the bias error in conventional centroid algorithms used for slope measurement in Shack-Hartmann wave-front sensors is presented for the first time. It is found that the bias can be as the same order of magnitude of the centroid error, especially at moderate and high SNR ratios, typically the bias error is larger than the noise error for SNR larger than 10, except for the solar case where it becomes important for SNR larger than 200.

No centroid method reduces both the bias and noise error terms in conventional correlation methods. A window shift method is proposed based on the anti-symmetric nature of the bias. It works by sampling the sub-aperture image $K$ times, at the same resolution, but shifted by a sub-pixel step, with size function of $K$. The obtained $K$ shifts are then averaged out, significantly reducing the bias. The window 
shift method is studied as a function of image type, centroid algorithm, SNR and $K$ sampling factor. It is found that it robustly reduces the bias by a factor of $\sim 7$ to values of $\lesssim 0.02$ pix. The computational cost of the algorithm is optimized by obtaining the correlation in two steps: a) large region based coarse search; b) small region based $C_{5}$ fine search. It ranges between a factor of 1.4 to 3.6 of conventional approaches.

The window shift method can be applied to other algorithms which work similar to the cross-correlation algorithm such as square difference function, absolute difference function and square of the absolute difference function (Löfdahl 2010). The square difference function is especially important for the solar type of images as it gives a significantly smaller random error and more anti-symmetric pattern of systematic error (Löfdahl 2010). However, the systematic error values are larger in a comparison to the cross-correlation. The proposed method would be of relevance for the square difference function to reduce its systematic error by using its consistent anti-symmetric pattern.

Further developments are the study of the window shift algorithm for sub-aperture images that have a sampling smaller than the critical sampling and for Shack-Hartmann devices with a small number of apertures, such as those used for fast tip-tilt correction or pupil tracking.

\section{ACKNOWLEDGEMENTS}

We are grateful to Lancelot, J. P and Akondi, V for commenting and proof reading of the manuscript. We thank the referee for her/his constructive and insightful report. This research was partially supported by Fundação para a Ciência e a Tecnologia (contracts PTDC/CTE-AST/116561/2010, SFRH/BD/52066/2012, UID/FIS/00099/2013) and the European Commission (EC) (Grant Agreement 312430). MC wishes to acknowledge A*MIDEX project (no. ANR-11IDEX-0001-02) funded by the French Government programme "Investissements d'Avenir", managed by the French National Research Agency (ANR).

\section{REFERENCES}

Akondi, V. \& Vohnsen, B. 2013, Ophthalmic and Physiological Optics, 33, 434-443

Bailey, D. G. 2003, in Proceeding of Image and Vision Computing New Zealand, 414-419

Basden, A. G., Chemla, F., Dipper, N., et al. 2014, MNRAS, 439, 968

Booth, M. J. 2014, Light: Science \& Applications, 3, e165

Burns, S. A., Elsner, A. E., Chui, T. Y., et al. 2014, Biomedical Optics Express, 5, 961

Dai, G.-M. 1996, Journal of the Optical Society of America A, 13,1218

Feautrier, P., Gach, J.-L., \& Bério, P. 2016, Proc. SPIE, 9907, 990715

Finger, G., Baker, I., Alvarez, D., et al. 2014, Proc. SPIE, 9148, 914817

Gratadour, D., Mugnier, L. M., \& Rouan, D. 2005, A\&A, 443, 357

Gratadour, D., Gendron, E. \& Rousset, G. 2010, J. Opt. Soc. Am. A 27, A171

Gui, L., \& Wereley, S. T. 2002, Experiments in Fluids, 32, 506
Löfdahl, M. G. 2010, A\&A, 524, A90

Malacara, D. 2007, Optical shop testing (John Wiley)

Nobach, H. \& Honkanen, M. 2005, Experiments in Fluids, 38, 511

Poyneer, L. A. 2003, Appl. Opt., 42, 5807

Rais, M., Morel, J. M., Thiebaut, C., Delvit, J. M.\& Facciolo, G. 2016, In Journal of Physics: Conference Series, 756, 1

Rigaut, F. \& Van Dam, M. 2013, Third AO4ELT Conference Adaptive Optics for Extremely Large Telescopes, 13173

Robert, C., Michau, V., Fleury, B., Magli, S., \& Vial, L. 2012, Optics Express, 20, 14, 15636

Schreiber, L., Foppiani, I., Robert, C., et al. 2009, MNRAS, 396, 3,1513

Schreier, H. W., Braasch, J. R., \& Sutton, M. A. 2000, Optical Engineering, 39, 11

Sidick, E. 2013, Appl. Opt., 52, 26, 6487

Thomas, S., Fusco, T., Tokovinin, A., et al. 2006, MNRAS, 371, 1,323

Thomas, S. J., Adkins, S., Gavel, D., Fusco, T., \& Michau, V. 2008,MNRAS, 387, 173

Townson, M. J., Kellerer, A., \& Saunter, C. D. 2015, MNRAS, 452, 4022

Tyson, R. K. 2015, Principles of adaptive optics, 4th Edition, CRC Press

Vijaya Kumar, B. V. K., Dickey, F. M., \& Delaurentis, J. M. 1992, Journal of the Optical Society of America A, 9, 5, 678

Wöger, F. \& Rimmele, T. 2009, Appl. Opt., 48,1, A35

This paper has been typeset from a $\mathrm{T}_{\mathrm{E}} \mathrm{X} / \mathrm{LAT}_{\mathrm{E}} \mathrm{X}$ file prepared by the author. 